Annuaire suisse de politique de développement

$17 \mid 1998$

Propriété intellectuelle : quels enjeux pour les pays en développement ?

\title{
Brevets dans le secteur pharmaceutique : point de vue de l'industrie pharmaceutique suisse
}

Brian A. Yorke et Philip W. Grubb

\section{(2) OpenEdition}

\section{Journals}

Édition électronique

URL : http://journals.openedition.org/aspd/742

DOI : $10.4000 /$ aspd. 742

ISSN : 1663-9669

Éditeur

Institut de hautes études internationales et du développement

Édition imprimée

Date de publication : 1 avril 1998

Pagination : 105-116

ISSN : 1660-5934

Référence électronique

Brian A. Yorke et Philip W. Grubb, «Brevets dans le secteur pharmaceutique : point de vue de l'industrie pharmaceutique suisse », Annuaire suisse de politique de développement [En ligne], 17 | 1998, mis en ligne le 22 juillet 2012, consulté le 08 septembre 2020. URL : http://journals.openedition.org/ aspd/742 ; DOI : https://doi.org/10.4000/aspd.742 


\section{BREVETS DANS LE SECTEUR PHARMACEUTIOUE: POINT DE VUE DE L'INDUSTRIE PHARMACEUTIQUE SUISSE}

BRIAN A. YORKE* ET PHILIP W. GRUBB**

A U COURS des dernières années, la protection par brevets des médicaments a été considérablement renforcée, en particulier dans les pays en voie de développement, ce qui a été très favorablement accueilli par l'industrie pharmaceutique et tient en grande partie à l'adoption de l'ADPIC dans le cadre du GATT (Accord général sur les tarifs douaniers et le commerce).

Dans cet article, nous nous intéressons aux modifications de la loi sur les brevets rendues nécessaires par l'ADPIC, en étudiant en particulier la situation dans les pays en voie de développement, et passons en revue les progrès de la mise en œuvre de l'ADPIC.

Le GATT a été signé en 1948 pour régler les questions commerciales multilatérales. Le dernier cycle des négociations du GATT, l'Uruguay Round, s'est terminé en avril 1994 et a abouti à la création de l'OMC (Organisation mondiale du commerce), qui est devenue opérationnelle en janvier 1995. L'ADPIC (Accord relatif aux aspects de propriété intellectuelle qui touchent au commerce y compris le commerce des marchandises de contrefaçon) a été adopté comme partie intégrante de l'Acte final de l'Uruguay Round, et tous les pays qui ratifient le nouveau texte du GATT et deviennent membres de l'OMC doivent accepter les dispositions de l'ADPIC comme partie intégrante de l'acte.

L'ADPIC traite de nombreuses questions de propriété intellectuelle, dont les brevets, les marques, les indications géographiques, les dessins industriels, les circuits intégrés, la protection des droits d'auteur et du secret de fabrication ainsi que les dispositions générales concernant les principes de base, l'application et le règlement des litiges. Dans le commentaire suivant, nous nous concentrerons essentiellement sur les brevets et en particulier sur ce qui a trait aux médicaments.

\section{IMPORTANCE DU GATT/ADPIC}

\subsection{PRINCIPES DE BASE DE L'ADPIC}

Ils comprennent les principes de base du traitement des ressortissants et du traitement des nations les plus favorisées, c'est-à-dire que chaque membre ne doit pas accorder aux ressortissants des autres pays membres un traitement moins favorable que celui accordé à ses propres ressortissants et chaque membre doit accorder aux ressortissants de tous les pays membres les mêmes privilèges que ceux donnés aux ressortissants de n'importe quel pays membre. Ainsi, à

* Directeur, Service de la propriété intellectuelle de Novartis International AG.

** Conseiller, Service de la propriété intellectuelle de Novartis International AG. 
quelques exceptions près, cela signifie que les accords bilatéraux entre membres ne doivent plus être permis. Ces articles sont particulièrement importants car leur application, contrairement aux autres dispositions de l'ADPIC, ne peut pas être repoussée au-delà du $1^{\text {er }}$ janvier 1996 par chaque pays membre, et ce, quel que soit son stade de développement.

\subsection{DISPOSITIONS CONCERNANT SPÉCIFIQUEMENT LES BREVETS}

L'ADPIC exige des Etats membres de l'OMC l'introduction d'une forte protection par brevets, dont les principes fondamentaux sont les suivants :

- Les brevets doivent répondre essentiellement aux mêmes critères de brevetabilité que ceux énoncés dans la Convention européenne en matière de brevets pour tous les secteurs de la technologie, y compris les brevets de produits pour les médicaments. Les membres peuvent exclure de la brevetabilité certaines catégories d'inventions telles que les méthodes de traitements médicaux et, du moins pour le moment, les plantes et les animaux, mais il n'existe pas par exemple de clause stipulant que la prise d'un brevet pour un gène humain pourrait être exclue s'il répond aux critères habituels de nouveauté, d'activité inventive et d'applicabilité industrielle. Il ne faut pas oublier que si la Suisse devait approuver et appliquer la Genschutzinitiative (initiative populaire concernant la protection des gènes), cela serait une violation de ses obligations envers le GATT/ADPIC.

- Les droits en matière de brevets doivent être concédés et appliqués sans discrimination, que les produits brevetés soient conçus localement ou soient importés. Cela signifie qu'il ne sera plus possible de concéder des licences obligatoires ou d'invalider un brevet si le produit n'est pas fabriqué localement, aussi longtemps que le marché national sera suffisamment approvisionné par l'importation du produit fabriqué à l'étranger.

๖ Il est précisé que la contrefaçon comprend l'importation d'un produit breveté et l'utilisation, la vente ou l'importation du produit issu directement d'un procédé breveté. Dans quelle mesure le titulaire d'un brevet peut empêcher l'importation illicite de produits brevetés qu'il met sur le marché dans un autre pays, reste moins clair.

- Les licences obligatoires ne peuvent être autorisées que sous de strictes conditions. Il ne sera plus possible de concéder des licences obligatoires de façon automatique, par exemple pour toutes les inventions pharmaceutiques.

a L'application des droits en matière de brevets se fait sans discrimination envers le secteur de technologie, ce qui signifie non seulement qu'il n'est pas permis de concéder des licences obligatoires dans un secteur particulier tel que les médicaments, mais que d'autres formes de discrimination sont également interdites. Par exemple, des lois permettant ce qui autrement constituerait des actes de contrefaçon pendant la durée du brevet et permettant le lancement d'une copie générique d'un médicament le lendemain de l'expiration du brevet sont contraires aux principes de l'ADPIC.

- Un recours en justice doit être possible pour toute décision d'annulation d'un brevet. 
- La durée d'un brevet doit être d'au moins vingt ans à partir de la date de dépôt. Selon les dispositions transitoires de l'ADPIC, cela devrait également s'appliquer aux demandes de brevets en instance lors de la modification de la loi ainsi qu'aux brevets qui ont déjà été délivrés.

- Il faudrait une inversion de la charge de la preuve pour les brevets de procédés, c'est-à-dire que lorsqu'un nouveau produit est uniquement protégé par un brevet couvrant son procédé de fabrication, si le produit est contrefait, on suppose qu'il a été obtenu selon le procédé breveté, sauf si le contrefacteur peut prouver le contraire.

\subsection{PROTECTION DES INFORMATIONS NON DIVULGUÉES}

La disposition qui incite les membres à protéger les données d'enregistrement non divulguées contre l'utilisation commerciale déloyale, même si elle ne concerne pas directement les brevets, présente un intérêt particulier pour l'industrie pharmaceutique. De nombreux pays développés, y compris la Suisse, ne disposent pas actuellement d'une protection exclusive des données. D'autres pays lient la durée de la protection à celle du brevet, ce qui réduit considérablement la valeur de la protection et devrait être considéré comme contraire aux principes de l'ADPIC, étant donné que l'article se rapportant à cette disposition est complètement séparé de la section concernant les brevets. L'ADPIC ne stipule aucune durée de protection des informations, et l'industrie pharmaceutique propose une durée type à l'échelon mondial de dix ans à partir de l'enregistrement dans chaque pays.

\subsection{DISPOSITIONS POUR L'APPLICATION DES DROITS DE LA PROPRIÉTÉ INTELLECTUELLE (PI)}

Toute une section de l'accord de l'ADPIC est consacrée à l'application des droits de la propriété intellectuelle, y compris des brevets. Les membres sont dans l'obligation de disposer de procédures d'application qui soient efficaces, justes et équitables, sans être nécessairement coûteuses. Il doit exister une clause de sauvegarde comprenant le droit de représentation légale, le droit de commenter toutes les preuves sur lesquelles se base la décision et le droit d'examiner judiciairement les décisions administratives.

En matière de PI, les autorités judiciaires doivent pouvoir exiger la production de preuves et se prononcer concernant les coûts, les dommages et intérêts et la destruction ou la confiscation des produits contrefaits. Les juges doivent être à même d'adresser des injonctions préliminaires ou interlocutoires dans des cas précis, sans que le défenseur ne soit notifié. Les membres doivent adopter des procédures pouvant permettre aux autorités douanières, sur requête, d'empêcher l'importation de marques contrefaites ou de produits contrefaits protégés par le droit d'auteur (pas nécessairement toutefois de contrefaçons de brevets). Des sanctions pénales doivent être prononcées pour la contrefaçon délibérée de marques ou du droit d'auteur à une échelle commerciale; des sanctions pénales pour d'autres types de contrefaçons de la PI sont facultatives. 


\subsection{RÉSUMÉ}

Grâce à l'ADPIC, presque tous les pays du monde sont obligés de disposer de systèmes de brevets permettant de breveter des composés pharmaceutiques pour une durée d'au moins vingt ans, sans obligation de fabrication locale et sans concession routinière de licences obligatoires, l'importation d'un produit et la vente du produit d'un procédé étant clairement considérées comme des contrefaçons; et ils doivent disposer de normes précises pour l'application des droits en matière de brevets. Cela représente non seulement un grand pas en avant pour l'industrie pharmaceutique basée sur la recherche, mais fournit également une bonne occasion aux pays en voie de développement d'adopter les systèmes de la PI qui favoriseront la poursuite de leur développement économique.

Il est également important que le GATT dispose à présent de procédures significatives pour régler les litiges et pouvant être utilisées pour prendre des sanctions contre un membre qui déroge à ses engagements vis-à-vis de l'ADPIC. Selon l'industrie, il est regrettable que ces procédures de règlement des litiges ne servent pas à régler les questions concernant l'épuisement des droits afférents aux brevets. Selon cette théorie, les droits afférents aux brevets sont «épuisés » par la vente d'un produit breveté par le titulaire du brevet ou avec son consentement dans n'importe quel pays (même dans un pays où il n'y a pas de brevet!) et l'importation non autorisée de ces produits dans un autre pays ne peut pas être empêchée par l'existence d'un brevet dans ce pays. Une telle libre circulation des produits ne devrait être permise que dans une zone de marché commun où, contrairement à l'UE, il n'y a pas de distorsion du marché provoquée par les politiques de prix des gouvernements.

\section{MODIFICATIONS DES LOIS SUR LES BREVETS SUITE À L'ADPIC}

Le $1^{\text {er }}$ janvier 1995, presque tous les pays membres de l'OMC dans le monde disposaient de lois de propriété intellectuelle qui, d'une manière ou d'une autre, n'étaient pas conformes aux principes de l'ADPIC. De nombreux pays, qu'il s'agisse de pays en voie de développement ou de pays industrialisés, avaient des durées de brevets qui étaient ou pouvaient être inférieures aux vingt ans à partir du dépôt stipulés par l'ADPIC. Cela a pu être corrigé relativement facilement, bien que certains pays aient mis du temps à le faire. Dans le cas de certains pays tels que l'Inde, l'Argentine, le Brésil et la Turquie, la protection par brevets des médicaments faisait totalement ou pratiquement totalement défaut et des révisions majeures étaient nécessaires, pendant lesquelles on devait appliquer les "dépôts gelés des demandes" désignés black box filings (voir la section 3 ciaprès). Toutefois, même des pays industrialisés tels que les Etats-Unis, les pays de l'Union Européenne et le Japon disposaient de lois qui, de façon plus subtile, n'étaient pas conformes aux principes de l'ADPIC telles que les conditions pour l'octroi des licences obligatoires. Toutes ces lois, si elles ne sont pas déjà changées, doivent être mises en conformité avec les accords de l'ADPIC. Le calendrier des réalisations est le suivant. 


\subsection{DISPOSITIONS TRANSITOIRES DE L'ADPIC}

\section{$\square$ Délais pour l'application}

Aucun pays n'a été obligé de mettre ses lois en conformité avec les principes de l'ADPIC dans l'année qui a suivi la création de l'OMC, c'est-à-dire jusqu'au

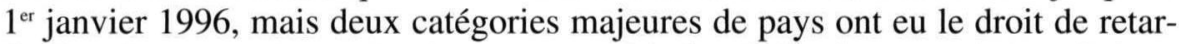
der les changements sur de plus longues périodes. Selon les accords transitoires, les pays en voie de développement peuvent retarder l'application des principes de l'ADPIC pendant encore quatre ans, c'est-à-dire jusqu'au $1^{\text {er }}$ janvier 2000 . Il en va de même pour les pays en train de passer d'une économie de planification centralisée à une économie de libre marché qui ont des difficultés à mettre en place un nouveau système de PI. Cela ne concerne pas par exemple la Hongrie, la Pologne et la République tchèque qui disposent de systèmes de brevets appropriés.

Les pays en voie de développement qui à partir du $1^{\text {er }}$ janvier 2000 ne disposeront toujours pas de protection des produits dans certains secteurs de la technologie (par exemple pour les médicaments) pourront retarder l'introduction de la protection des produits dans ces secteurs (mais ne pourront pas se soustraire aux autres obligations de l'ADPIC) pendant encore cinq ans, c'est-à-dire jusqu'au $1^{\text {er }}$ janvier 2005. Et les pays les moins développés qui sont membres de l'OMC auront le droit de retarder l'application de toutes les dispositions de l'ADPIC autres que les principes de base indiqués dans le paragraphe 1.1. ci-dessus jusqu'au $1^{\text {er }}$ janvier 2006 , et ce délai pourra même être prolongé sur requête spéciale.

2006 est encore loin, mais les pays les moins développés représentent un groupe bien défini (49 pays selon la liste officielle de l'ONU), dont le niveau de développement est tel qu'ils est peu probable qu'ils représentent des marchés importants même dans dix ans. Les entreprises pharmaceutiques ne peuvent pas déposer des brevets dans chaque pays et une amélioration de la protection par brevets dans les pays les moins développés a peu de chance d'avoir une importance pratique. Par contre, il n'y a pas de critère objectif concernant la définition d'un «pays en voie de développement» et un certain nombre de pays économiquement importants se sont effectivement déclarés «pays en voie de développement» dans le contexte de l'ADPIC, ce qu'ils n'auraient pas fait autrement.

\subsection{AJUSTEMENT DE LA DURÉE DES BREVETS}

L'ajustement de la durée des brevets pour la rendre conforme aux dispositions de l'ADPIC se fait relativement facilement et la majorité des Etats membres de l'OMC l'ont déjà fait, y compris l'Argentine, le Brésil, l'Indonésie et les Philippines, alors que la Malaisie n'a pas encore changé la durée de quinze ans à partir de la délivrance. La Corée a déjà changé sa loi dans ce sens: la durée était antérieurement la période la plus courte, correspondant soit à vingt ans à partir du dépôt, soit à quinze ans à partir de la publication pour l'opposition. La dernière possibilité a été à présent supprimée. Toutefois, le changement en Corée n'est effectif que depuis le $1^{\text {er }}$ juillet 1996. La nouvelle loi coréenne en matière de brevets a prolongé la durée des brevets en vigueur à partir de cette date; par contre, les brevets qui devaient expirer dans la première moitié de 1996 et 
auraient dû être prolongés ne seront plus en vigueur. Dans d'autres pays, par exemple en Argentine et au Brésil, la durée des brevets existants n'a pas été prolongée, et cela a été porté devant les tribunaux.

\subsection{LICENCES OBLIGATOIRES}

L'ADPIC stipule que ces types de licences doivent:

- être considérés en fonction de leurs propres valeurs;

- être concédés uniquement si une licence de durée normale a été requise et refusée;

- être de portée et de durée limitées;

口 être incessibles sauf avec l'entreprise;

口 servir à l'approvisionnement domestique plutôt qu'à l'exportation ;

- pouvoir être résiliés si les circonstances changent;

口 être soumis à une rémunération appropriée ;

- être soumis à un examen judiciaire.

Les licences obligatoires permettant l'exploitation d'un brevet dépendant ne peuvent être concédées que lorsque l'invention revendiquée dans le dernier brevet dépendant implique une avance technique importante de portée économique considérable et, lorsqu'elle est concédée, la licence obligatoire doit être incessible sauf lors de la cession du brevet dépendant. Lors de la révision effectuée fin 1995 par le groupe des 15 de la Commission européenne, aucun Etat membre de la CE ne disposait de lois tout à fait conformes à toutes ces dispositions; il est donc peu surprenant que les lois des pays en voie de développement soient souvent loin d'être parfaites. Mais il est clair que l'octroi discriminatoire de licences obligatoires pour les brevets pharmaceutiques doit être supprimé. Il est aussi préoccupant que certaines dispositions de l'ADPIC concernant les licences obligatoires ne soient pas applicables aux licences obligatoires concédées pour combattre les pratiques anticoncurrentielles. Il s'agit d'une lacune qui peut permettre d'échapper à ces dispositions sur simple intervention d'un organe administratif déclarant que la conduite du détenteur d'un brevet est anticoncurrentielle. Dans le pire des scénarios, un contrefacteur peut proposer un produit breveté à un prix inférieur, le détenteur du brevet qui essaie d'arrêter la contrefaçon est traité d'anticoncurrentiel et le contrefacteur obtient une licence obligatoire.

\subsection{INTRODUCTION DE LA PROTECTION DES PRODUITS POUR LES MÉDICAMENTS}

Cela s'avère être un point particulièrement délicat de l'application de l'ADPIC. Comme indiqué plus haut, les pays en voie de développement peuvent la retarder jusqu'en 2005, et peuvent recourir entre-temps aux black box filings «dépôts gelés des demandes» (voir plus bas). L'industrie pharmaceutique est alors en droit de se demander si des pays comme l'Argentine et la Turquie, qui n'ont pas encore introduit la protection des produits pour les médicaments, peuvent être considérés à juste titre comme pays en voie de développement. Tous les pays, y compris la Suisse, sont, après tout, toujours en phase de développement. 


\section{BLACK BOX FILINGS «DÉPÔTS GELÉS DES DEMANDES »}

Un concept important des dispositions transitoires de l'ADPIC en matière de protection des matières existantes concerne les black box filings. Ces dispositions sont toujours ignorées ou mal appliquées dans certains pays.

Lorsqu'à la date d'entrée en vigueur de l'accord de création de l'OMC, un membre ne dispose pas de protection par brevets pour les produits pharmaceu-

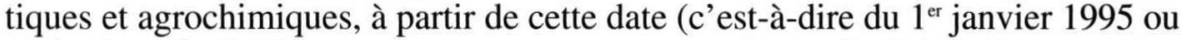
de la date d'adhésion de ce pays, si cela a lieu plus tard) il doit disposer d'un système permettant le dépôt des demandes pour ce genre d'inventions.

Les critères de brevetabilité stipulés par l'ADPIC doivent s'appliquer à ces demandes à partir de la date d'application de cet accord (c'est-à-dire la date indiquée plus haut, à partir de laquelle les obligations de la partie II section 5 de l'ADPIC s'appliquent à ce pays), comme si ces critères devaient être appliqués à la date de dépôt dans ce pays.

Ce système doit alors fournir une protection par brevets à partir de la délivrance pour le reste de la durée du brevet (c'est-à-dire vingt ans à partir de la date de dépôt), lorsque l'invention est brevetable selon les critères de l'ADPIC. En outre, un produit qui a bénéficié du système des black box filings doit bénéficier de droits exclusifs de commercialisation pendant cinq ans à partir de l'autorisation de mise sur le marché dans le pays concerné ou jusqu'à ce qu'un brevet du produit soit délivré ou rejeté, quel que soit le temps le plus court, à condition que, pour ce produit:

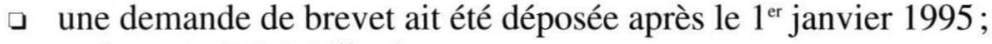

๑ un brevet ait été délivré;

- l'autorisation de mise sur le marché ait été obtenue;

tout cela dans un autre pays membre. Ces dispositions sont complexes et nécessitent la coopération des offices nationaux des brevets et des autorités de contrôle de la santé, si l'on veut qu'elles soient efficaces.

Ces dispositions compliquées désignées black box filings sont très importantes car, jusqu'à l'entrée en vigueur de nouvelles lois, elles constituent le seul moyen d'obtenir une protection par brevets des produits pharmaceutiques sur des marchés importants tels que l'Inde, le Pakistan, la Turquie et l'Argentine.

En considérant l'analyse ci-dessus, il ne fait aucun doute, même si cela n'est pas clairement exprimé, que de telles demandes pour des composés pharmaceutiques ne seront pas examinées (hormis peut-être un examen officiel pour établir que la demande peut bénéficier d'une date de dépôt) jusqu'à ce que le pays en question ait changé sa loi pour la rendre conforme aux dispositions de l'ADPIC concernant la brevetabilité des produits pharmaceutiques. Cela explique en fait pourquoi les dépôts de ces demandes ont été désignés «dépôts gelés». De plus, il est clair que tous les membres doivent disposer de tels dépôts à partir du

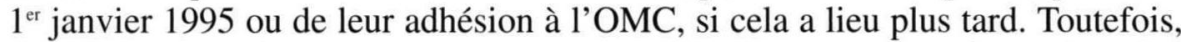
certains pays ne font pas du tout rentrer de telles demandes dans le système des black box filings ou les en retirent bien trop tôt.

L'Inde est un bon exemple parmi ces pays. Il existe dans ce pays un sérieux conflit entre les hommes politiques, à la fois dans l'ancien et dans l'actuel gouvernement de l'Inde, qui désirent l'ouverture de l'économie indienne sur le 
monde extérieur et considèrent la participation à l'OMC comme un élément important de cet objectif, et les personnes bénéficiant d'un grand soutien populaire qui souhaitent maintenir l'ancien protectionnisme et bloquer toute modification de la loi sur les brevets, laquelle est tout à fait inappropriée. L'Inde a donc ratifié les accords du GATT le 31 décembre 1994 par un décret gouvernemental, sans chercher l'accord du Parlement indien, et en a profité pour modifier la loi sur les brevets par une ordonnance d'amendement des brevets permettant ainsi les black box filings.

L'ordonnance est ensuite tombée en désuétude et le projet de loi qui devait la remplacer n'a pas obtenu l'accord du Parlement, ce qui fait que même si les black box filings sont toujours acceptés, ils ne sont plus reconnus officiellement et leur statut légal n'est pas clair. Cette ambiguïté persiste, car une législation d'application spéciale est nécessaire pour permettre les black box filings. Ce n'est pourtant pas le cas, que la constitution indienne fasse entrer en vigueur automatiquement ou non le traité du GATT. Les dépôts peuvent être faits sous forme de dépôts normaux dans le cadre de la loi existante et peuvent être ensuite maintenus en instance par une mesure purement administrative, cela jusqu'à la modification de la loi, si tant est qu'elle le soit un jour. Les dispositions des droits exclusifs de commercialisation (DEC) prévus par les accords transitoires de l'ADPIC ont soulevé de fortes objections et bien que, contrairement aux principes de l'ADPIC, l'ordonnance concernât l'octroi d'une licence obligatoire et la fixation des prix pour les DEC, des propositions ont été faites pour affaiblir encore plus leur effet. Le débat porte actuellement essentiellement sur l'application des dispositions transitoires en Inde et rien ou presque rien n'a été fait en vue d'une révision complète de la loi indienne sur les brevets pour la rendre conforme aux principes de l'ADPIC. Des procédures judiciaires sous l'égide de l'OMC, qui ont été lancées par les Etats-Unis et acceptées par 1'Inde, sont actuellement en cours. La commission de règlement des différends de l'OMC a récemment pris des décisions contre l'Inde, qui peut faire appel.

La loi sur les brevets de l'Inde est sans aucun doute la pire du monde - du moins pour un pays économiquement important. Pour les inventions pharmaceutiques, il n'est possible de breveter qu'un procédé de fabrication d'un produit, bien que les nombreux chimistes indiens compétents puissent facilement trouver de nouveaux procédés; la durée est limitée à sept ans, c'est-à-dire que le brevet expire avant que le produit ne puisse être introduit en Inde; et, dans le cas où il subsiste une certaine durée du brevet, il y a des licences obligatoires automatiques et une redevance de $4 \%$ sur le prix de gros; une «redevance» qui est en fait une forme déguisée de confiscation.

Les activistes indiens sont mécontents lorsqu'une entreprise occidentale brevette (mais bien sûr pas en Inde) une nouvelle composition dérivée de l'arbre indien margousier. Ils devraient plutôt se demander pourquoi aucune entreprise indienne n'investit dans la recherche de nouveaux dérivés des médecines indiennes traditionnelles. La réponse est simple: ils ne peuvent pas les breveter en Inde et n'importe quelle autre entreprise est libre de copier. Il est temps que l'Inde se défasse de sa mentalité postcoloniale et rejoigne les autres pays asiatiques qui comprennent que le développement économique va de pair avec une forte protection par brevets. 
Jusqu'à une date encore récente, le Pakistan a également refusé d'accepter les black box filings sous prétexte de ne pas disposer de législation d'application. Toutefois, un projet de loi pour la mise en place des black box filings a été récemment arrêté et s'appliquera rétroactivement aux dépôts effectués depuis le $1^{\text {er janvier } 1995 .}$

La Turquie a manifesté différemment sa non-soumission aux dispositions de l'ADPIC concernant les black box filings. Elle accepte les dépôts pour des composés pharmaceutiques, mais bien que la loi n'ait pas encore été amendée, les demandes ne sont pas traitées selon le système des black box filings, mais sont soumises aux mêmes recherches excessivement coûteuses que les demandes des autres secteurs de la technologie. Toutefois, cela a au moins un effet positif, car lorsque la loi aura été changée, il ne restera pas beaucoup de demandes non examinées. A l'origine, la Turquie a même proposé de retarder de cinq ans la délivrance des brevets de procédés pour les médicaments et de dix ans celle des brevets de produits. Cela correspondait peu à l'application déployée par la Turquie pour rejoindre l'union douanière en vue de devenir ensuite un membre à part entière de l'UE, mais il semble à présent que la Turquie pourra adhérer à la Convention européenne des brevets au plus tard en 1999.

Le Brésil représente une réussite dans la saga du GATT-ADPIC. Dès le départ, les black box filings ont été acceptés sans difficulté et une nouvelle loi sur les brevets accordant la protection aux produits pour les médicaments est entrée en vigueur le 15 mai 1997. La nouvelle loi est tout à fait conforme aux dispositions de l'ADPIC, sauf qu'elle exige encore une fabrication locale. Sous deux aspects, elle va en fait plus loin que ce qui est stipulé par l'ADPIC, en fournissant un système de protection transitoire et en permettant également d'empêcher les importations parallèles. Un point négatif est que la durée de vingt ans instaurée par l'ADPIC n'a pas encore été étendue aux brevets déjà délivrés.

Il en va malheureusement autrement avec l'Argentine. L'exécutif s'est montré en général plutôt favorable à une amélioration de la protection par brevets, mais le législatif a été fortement influencé par le groupe de pression local de l'«industrie pharmaceutique», c'est-à-dire un petit groupe d'entreprises lucratives qui gagnent de l'argent simplement en vendant des médicaments génériques ou des copies de médicaments brevetés qui sont importés d'autres pays tels que l'Inde. Ces entreprises apportent peu de valeur ajoutée dans le pays.

Le résultat est qu'après une succession sans fin de nouvelles lois, de veto présidentiels, de décrets, de «projets de lois correctives», etc., une nouvelle loi a été promulguée et est entrée en vigueur en janvier 1997. Non seulement cette loi retarde l'introduction de la protection des produits jusqu'au $1^{\text {er }}$ janvier 2001 (en d'autres termes, l'Argentine doit être considérée comme un pays en voie de développement), mais elle est contraire à l'ADPIC à bien d'autres égards. Les black box filings peuvent être faits, mais il n'y a pas de disposition d'exclusivité de commercialisation, ce qui est particulièrement important si, comme dans le cas présent, l'application de la protection des produits se fera au terme d'un long délai. 


\section{DÉLIVRANCE ET APPLICATION DES BREVETS}

Il est peu utile d'avoir sur le papier une forte loi sur les brevets, sauf si les demandes de brevets parviennent à la délivrance et si les brevets résultants peuvent être appliqués. De nombreux pays en voie de développement ont récemment amélioré leurs lois sur les brevets, ce qui implique souvent un examen de fond de la nouveauté et de l'activité inventive des demandes de brevets. Cela place ces pays dans une position difficile: en raison de l'amélioration de leurs lois, leurs offices des brevets reçoivent beaucoup plus de demandes qu'avant, mais ils ne disposent pas du personnel technique et des infrastructures nécessaires pour examiner les demandes de brevets comme semblent l'exiger ces lois. Il ne faut pas sous-estimer le problème; pour être capable d'effectuer un examen de fond dans tous les domaines techniques, un office des brevets doit disposer de nombreuses personnes techniquement qualifiées et d'énormes ressources en littérature scientifique et en brevets, avoir accès aux bases électroniques de données, etc. Il n'existe probablement pas plus de dix offices des brevets dans le monde qui en soient capables. Non seulement cela dépasse de beaucoup les moyens de la plupart des pays en voie de développement, mais cela serait un gaspillage absurde de leurs ressources s'ils devaient réaliser à nouveau un travail, qui, dans la plupart des cas, a déjà été effectué par l'office des brevets américain, européen ou japonais.

Les organisations du type OEB (Office européen des brevets) et OMPI (Organisation mondiale de la propriété intellectuelle) à Genève aident les offices des brevets des pays en voie de développement à traiter le flux des nouvelles demandes, si possible par des moyens évitant un examen technique indépendant, et ils délivrent des brevets s'appuyant sur l'acceptation de brevets correspondants dans un des offices des brevets importants ou lorsque le pays en question a adhéré au Traité de coopération en matière de brevets (PCT), sur la base du rapport d'examen préliminaire international.

L'application nécessite des cours de justice compétentes dont les juges connaissent les lois sur les brevets; mais plus nécessaire encore est un environnement légal considérant la contrefaçon en propriété intellectuelle comme quelque chose que l'on doit fortement décourager par des amendes élevées, et non pas comme une industrie locale en croissance dont les activités devraient être ignorées ou même encouragées.

Depuis 1992, le Mexique dispose par exemple d'une excellente loi forte en matière de brevets, mais jusqu'à une date encore récente, aucun brevet n'avait été délivré, car il n'existait aucun règlement d'application. Cela est actuellement en train d'être corrigé et des brevets sont délivrés, mais leur application face aux contrefacteurs locaux se révèle extrêmement difficile.

Cela constitue aussi un sérieux problème en Chine qui, bien que disposant d'une loi sur les brevets parfaitement appropriée, n'a pas pour tradition de résoudre les litiges devant les tribunaux. Bien que les litiges relatifs aux brevets puissent être transmis à la Cour du peuple, la majorité d'entre eux sont réglés par une procédure administrative auprès de l'autorité administrative chargée des brevets. Il s'avère très souvent que l'entreprise de contrefaçon est la propriété de l'autorité administrative locale ou d'un autre corps de l'Etat, ce qui rend peu probable la mise en route d'une action efficace pour mettre fin à la contrefaçon. 


\section{AMENDEMENTS POSSIBLES À L'ADPIC}

L'article 27.3 (b) permet aux membres d'exclure les plantes et les animaux de la brevetabilité, à condition que les variétés de plantes puissent être protégées par des brevets ou par des formes spéciales de protection telles que les Droits des phytogénéticiens. Ce sous-paragraphe doit être révisé quatre ans après l'entrée en vigueur de l'accord de création de l'OMC, c'est-à-dire quatre ans après le

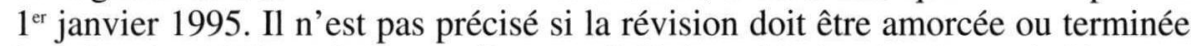

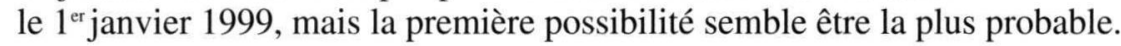

Du point de vue de l'industrie, cette révision doit strictement se limiter à savoir si l'exclusion des plantes et des animaux de la brevetabilité doit continuer à être permise, et on espère qu'après la "période de grâce» de quatre ans, les membres seront priés d'accorder une protection par brevets aux organismes vivants multicellulaires (il n'en demeure pas moins que les micro-organismes doivent être brevetables). Toutefois, de nombreuses organisations non gouvernementales et environnementales ainsi que certains pays en voie de développement tels que l'Inde souhaitent utiliser cela comme «point de départ» pour renégocier des sections entières de l'ADPIC. Il est fait référence en particulier au paragraphe précédent, l'article 27.2, qui déclare que les membres peuvent exclure de la brevetabilité «les inventions dont l'exploitation commerciale doit être interdite à l'intérieur de leur territoire pour respecter l'ordre public ou la moralité, y compris la protection de la vie ou de la santé humaine, animale ou végétale ou pour éviter des nuisances graves à l'environnement », et des tentatives sont faites pour associer l'accord ADPIC avec la Convention sur la biodiversité. Les forces anti-brevets espèrent ainsi être capables de faire modifier l'ADPIC pour refuser la brevetabilité par exemple des gènes humains (jugée «immorale»), de tout ce qui a trait aux examens des plantes ou des micro-organismes pour trouver des composés utiles («biocontrefaçon») et de tout ce qui pourrait avoir des effets néfastes sur l'environnement.

Premièrement, ni l'article 27.2, ni aucune autre partie de l'ADPIC autre que l'article 27.3 (b) ne sont soumis à une révision obligatoire.

Deuxièmement, la Convention sur la biodiversité concerne un ensemble de lois complètement différent de celui de l'ADPIC. Elle part du principe que les Etats ont des droits souverains sur leurs ressources biologiques et ses objectifs sont la conservation de la diversité biologique, son utilisation durable et le partage des bénéfices par accès aux ressources et au transfert de technologie (article 1). L'ADPIC, pour sa part, exige des Etats la protection de la propriété intellectuelle qui est reconnue comme un droit privé. Les deux principes sont parfaitement compatibles l'un avec l'autre, et aucun ne doit être appliqué pour contrecarrer les objectifs de l'autre. Les dispositions de la convention en matière de transfert de technologie stipulent en particulier que l'accès aux ressources et le transfert de technologie doivent être réalisés en des termes qui reconnaissent et vont dans le sens d'une protection appropriée et efficace des droits de la propriété intellectuelle (article 16.2). Les parties sont supposées s'assurer que les droits de la propriété intellectuelle qui sont soumis à la législation nationale et à la loi internationale (par exemple à l'ADPIC) soutiennent et ne sont pas contraires aux objectifs de la convention (article 16.5). Pour l'industrie, une forte protection par brevets est un encouragement au transfert des technologies. 
Troisièmement, la possibilité d'exclusion pour des motifs d'ordre public ou de moralité ne signifie pas que chaque groupe de pression a le droit d'empêcher la délivrance de brevets pour des inventions qu'il désapprouve. L'exclusion de la brevetabilité n'est possible que lorsque l'exploitation commerciale de l'invention «doit être empêchée». La brevetabilité n'est pas systématiquement exclue, même lorsque l'exploitation de l'invention est interdite par la loi intérieure du pays, et par conséquent, lorsque l'exploitation commerciale est légale, des brevets peuvent être délivrés. 